\title{
nature
} biotechnology

\section{Boldness, but with realism}

T: he US Biotechnology Industry Organization has joined forces with two philanthropic foundations-the Rockefeller Foundation and the Bill and Melinda Gates Foundation-to form a not-for-profit group dedicated to solving the business problems that biotech companies face in creating products for developing countries. The BIO Ventures for Global Health (BVGH) initiative will not fund research. It hasn't got enough money. BVGH was launched with only $\$ 1$ million from Gates and $\$ 100,000$ from Rockefeller. Instead, the program aims to point biotech R\&D more directly at developing world issues. The focus of the project is likely to be in infectious diseases, particularly those that fall outside the relatively well supported areas of AIDS (e.g., International AIDS Vaccine Initiative), tuberculosis (WHO Stop TB) and malaria (Medicines for Malaria Venture). Wendy Taylor, its executive director, sees BVGH as "a bold experiment."

It is commendably daring to conceive that biotech and the developing world can be allies against the scourge of disease. It is bolder still to propose a specific mechanism that can serve as a starting point for committed companies. However, while we applaud the initiative in its general aims, 'experiments,' no matter how bold, are, frankly, never going to solve anything anywhere, let alone in the developing world. As with any project, one needs at the very least to consider not only the market, but the means of reaching the market.

Undeniably there is a demand for better treatment for infectious disease. Life expectancy in the world's 42 poorest countries averages just 51 years, compared with 70 years or more in developed nations. One of the reasons for this is that people in sub-Saharan Africa, Asia and South America are burdened with diseases such as malaria, tuberculosis, hookworm, sleeping sickness, schistosomiasis and cholera.

On the supply side, biotech companies are focused almost exclusively on rich-world ailments like cancer and obesity. Currently, less than $10 \%$ of the industry's current research funding is aimed at diseases that account for $90 \%$ of the world's disease burden. So, it can be concluded, the supply side needs some improvement.

BVGH has several constructive strategies for tipping the balance. It will help companies adapt their business plans to include opportunities in products relative to the developing world. It will also help companies navigate through the funding initiatives of international agencies such as WHO or the UN that have little experience in dealing with biotech companies. The firms could access $\$ 4$ million to $\$ 15$ million per product for manufacture and early clinical testing. So far, so good.

But then there is a big problem. In order to get biotech companies fired up about developing world opportunities, they must believe the projects ultimately can succeed. Making this case forcefully is more difficult now than before the XV International AIDS conference in Bangkok last month. In the build-up to that meeting, it was revealed that the anti-retroviral drugs from Western companies were still not getting anywhere near patients in developing nations. This was not because the companies failed to supply them (as some AIDS pressure groups had feared) or that governments in poor countries would create a parallel market in the drugs (as the pharma companies had feared) but, more simply, that there was insufficient infrastructure to get the drugs to the patients that desperately need them. The demand and supply were in close proximity, but without medical staff or a system for distributing the drugs, the two didn't meet.

If BVGH did work, the benefits both to patients in developing countries and to the image of post-Waksal biotech would be immense. But without a careful and detailed consideration of the mechanisms by which the treatments can reach the patients, all the vision, support and good intentions in the world will not change a thing.

\section{Expose your clinical thinking}

The momentum that is gathering behind a call for an international registry of clinical trials is still being largely resisted by industry groups. Impending class action suits over the juvenile use of GlaxoSmithKline's Paxil (paroxetine $\mathrm{HCl}$ ) should keep the topic in the news. The major argument against such a registry is that to disclose a clinical trial is to give valuable commercial information to your competitors. Telling the world that one's drug trial has been halted or discontinued tells your competitors at least two things: that they shouldn't do the same as you, and that there is still a commercial opportunity to try some other solution in this area.

However, it is not always smart to keep quiet about clinical trials, especially for young companies. Putting experimental compounds into human trials represents a coming of age: it generates kudos that alters the level of attention and respect from investors, collaborators and competitors.

But why not go much further? As a young company, why not give away as much clinical information as possible as soon as possible. Generally speaking, early-stage biotech companies are not the citadels of clinical practice. By exposing a planned clinical approach to the widest possible scrutiny, companies may receive a good deal of feedback from clinicians who know why that approach may be flawed or even doomed.

Biotech companies might be able to convince an investor or two into believing that its clinical outcomes will be positive. But if the drug is wrong or the protocol is wrong, it will not fool the patients into getting better (or getting worse more slowly). For a biotech company running on lean cash reserves, it might be a setback to terminate a trial as soon as it has begun, but it could be terminal to wait until that trial is complete. 